\section{A study of group reminiscence therapy and emotional intelligence among elderly members}

\author{
Azam Bazooband, \\ Abdolvahab Baghbanian, ${ }^{2}$ Ghazal Torkfar ${ }^{3}$ \\ 'Department of Management, Babol \\ Branch, Payam Noor University Babol, \\ Iran; 'Faculty of Health Sciences, \\ University of Sydney, Australia; ${ }^{3}$ Menzies \\ Centre for Health Policy, School of Public \\ Health, University of Sydney, Australia
}

\section{Abstract}

This study aims to investigate the effects of group reminiscence therapy on elderly's emotional intelligence. A quasi-experimenta research with a pre-test-post-test control group was conducted in July 2015, with a sample of 40 elderly members referring to an (anonymous) Community Center in the city of Shiraz, Iran. A predesigned instrument, i.e., the Emotional Intelligence Questionnaire developed by Schering, was applied to collect data. SPSS Statistics v. 22.0 (Released 2013; IBM Corp. Armonk, NY, USA) was used to analyze data, with a $95 \%$ confidence level and a measurement error of $\alpha=0.05$. Hypothesis tests were mainly conducted to examine whether group reminiscence therapy correlates with emotional intelligence among the respondents. Findings revealed that the applied intervention i.e., group reminiscence therapy significantly associates with various dimensions of emotional intelligence including self-awareness, self-control, self-motivation, empathy and social skills in the older adults within the experiment group; i.e., the mean scores of the variables for the post-test administered on the experimental group were significantly higher than those on the control group. Group reminiscence therapy has the potential to enhance emotional intelligence in the elderly by helping them control their thoughts and emotions and learn problem-solving skills.

\section{Introduction}

In many cases, the elderly are one of the most vulnerable groups of our communities with specific physical, psychological and social health and welfare-related needs. Aging and associated chronic diseases often lead to a rise in frailty, dependency and disability of older adults within a society.
The aging phenomenon is the process of becoming older over time, which brings about physiological and social changes; however, an appropriate and adequate measure has not been developed in line with these changes to promote the physical, mental and/or social health of the elderly. In particular, limited scales exist to measure cognitive and mental disorders that overshadow the lifestyle of older adults relative to their physical disorders over time. ${ }^{1}$

Individuals gradually lose their physiological and social skills due to ageing. Although this decrease in performance status does not necessarily mean their dependency in the elderly, it has numerous effects on the degree of vulnerability among this population group. ${ }^{2}$ Cognitive disorder, for example, is one of the most common problems of the ageing with a wide spectrum of complications. About $35 \%$ of older adults have different levels of cognitive disorders with Alzheimer and dementia being the most progressive ones among them. ${ }^{3}$ There is evidence to suggest that cognitive therapy contributes to improve capabilities and cognitive function and memory restoration of people with Alzheimer's disease and dementia. ${ }^{4}$ Group, verbal and non-verbal reminiscence therapy in the elderly is one such therapy that can reduce cognitive and depressive symptoms commonly associated with Alzheimer and dementia. ${ }^{5,6}$ Group reminiscence therapy is a non-pharmacological intervention that aims to target cognitive functioning, and behavioral and psychological symptoms of co-occurring diseases associated with ageing including dementia. ${ }^{5,6}$ It is a kind of interpersonal relationship that offers the elderly or people with cognitive disorders an opportunity to practice social skills and establish mutual interactions with their environment including peers through expressing their feelings. ${ }^{7}$ Group reminiscence therapy has been found as an effective intervention to improve cognitive function in patients diagnosed with depression including dementia and in particular it has the potential to increase the effect of anti-dementia medications in the elderly prone to Alzheimer's disease when they come together. ${ }^{8}$

Emotional intelligence (EI) is defined as the ability to recognize, understand and manage our own emotions and the emotions of others. It is the capacity to be aware of, control, and express one's emotions, and to manage interpersonal relationships judiciously and empathetically. It is an important success factor that makes individuals with average intelligence quotient (IQ) reach great achievements. According to Goleman, ${ }^{10}$ any deficiencies in EI can bring damages and lead to problems in married life, childrearing, or even the physical condition of an individual. Lack of EI can subdue thought and disturb the professional status of people. The important point is that $\mathrm{EI}$ is not fixed at birth and it can be developed or sup-
Correspondence: Azam Bazooband, Department of Management, Babol Branch, Payam Noor University Babol, No 203, 66 St, 66/4 Alley, Pasdaran Blvd., P.0. Box 7184958975, Shiraz, Iran. Tel.: +98.9303306158.

E-mail: azam.bazooband@gmail.com

Key words: Community-aged center; elderly; emotional intelligence; group reminiscence therapy; older adults; Shiraz-Iran.

Conflict of interest: the authors declare no potential conflict of interest.

Acknowledgments: we would like to acknowledge the individuals who kindly donated their time and energy to participate in this study. We also thank the colleagues who provided insight and expertise towards implementation of this research even though they may not agree with all of the interpretations/conclusions of this paper.

Received for publication: 11 April 2016

Revision received: 6 June 2016.

Accepted for publication: 4 July 2016 .

This work is licensed under a Creative Commons Attribution-NonCommercial 4.0 International License (CC BY-NC 4.0).

(C) Copyright A. Bazooband et al., 2016

Licensee PAGEPress, Italy

Geriatric Care 2016; 2:5927

doi:10.4081/gc.2016.5927

pressed over time. ${ }^{10}$ IQ helps people be more successful in the fields of leadership and decision-making. EI can contribute to an increase in academic achievement through the features of emotional perception, emotional facilitation and emotional management as well as mechanisms to predict an increase in leadership power and to strengthen effective coping strategies. ${ }^{11}$ According to research findings, it can be argued that EI can provide for the improvement of social relationships by strengthening mental health, empathy with others, social compromise, emotional wellbeing, life satisfaction, and reduction in interpersonal problems. In addition, emotional perception, emotional facilitation, and emotional management are effective in improving social relationships through mechanisms of prediction, prevention, increased surveillance, and reinforced coping strategies which lead to the belief that EI (academic, occupational, and interpersonal) can be taught and modified in various social contexts with personal and social benefits as it develops. Moreover, it is claimed that EI plays a major role in determining life outcomes that go beyond the capabilities of general intelligence and personality factors. ${ }^{12}$

Today, patients of any age with various medical and psychological diagnoses can benefit from group therapy as a treatment for 
mental health. One of the short-term groups is reminiscence in group that is used in all age groups especially among the elderly to enhance their mental health. Reminiscence is an overview of life days spent. The benefits of reminiscence include the clarification of the sense of self in relation to others, reconstruction of the events recounted, restatement of previously used problem-solving strategies, and preparation for death. ${ }^{13}$

Despite the extensive use of reminiscence intervention across the world and its ease and low costs, it is not common in Iran with an increasing number of older adults asking for care. There have also been fewer attempts to investigate its effects on EI. Since a small number of studies have been conducted in terms of the effects of group reminiscence on EI in the elderly, the purpose of this study was to investigate the effects of group reminiscence on EI in the elderly members referring to a Community Center in the city of Shiraz.

To select the conceptual model as well as appropriate measurement tools (questionnaires), previous research studies done on group reminiscence and EI were reviewed and analyzed. So far, different scholars have proposed various models and developed different measurement tools (questionnaires) in this respect. In this study, the model of emotional intelligence developed by Goleman was used. This model, now widely recognized, does not mean that when a person has a high intellectual quotient or IQ, he or she does not necessarily have high EI. Being intelligent is a privilege, but not a guarantee of success in life and interpersonal as well as social relationships. In Goleman's model, five key areas of EI are examined: i) understanding of your own emotions and feelings, self-awareness; ii) management of your own emotions and feelings, selfmanagement; iii) self-motivation; iv) recognition and understanding of emotions and feelings of others, other-awareness; and v) management of relationships with others, othermanagement. ${ }^{14}$

\section{Materials and Methods}

The overall form of the conceptual model in this study according to the above explanations and the following hypotheses will be explained below.

According to this conceptual model (Figure 1), the following hypotheses can be proposed:

- Main hypothesis: interventions in the form of group reminiscence therapy have effects on emotional intelligence in the elderly.

- Secondary hypotheses: i) interventions in the form of group reminiscence therapy have effects on self-awareness in the elderly; ii) interventions in the form of group reminiscence therapy have effects on selfcontrol in the elderly; iii) interventions in the form of group reminiscence therapy have effects on self-motivation in the elderly; iv) interventions in the form of group reminiscence therapy have effects on empathy in the elderly; v) interventions in the form of group reminiscence therapy have effects on social skills in the elderly.

In terms of methodology, this study was a quasi-experimental research with a pre-testpost-test control group. Among all elderly members of a Community Center in Shiraz, those who had the least skills in reading and writing and were able to start eye contact and verbal communications, also who had no chronic psychiatric disease, no hospitalization history and no emotional crisis experimented within the last 3 months were considered in this study. In order to make sure about the homogeneity of the sample considered, in the beginning the cognitive status of seniors was assessed through the mini mental state examination questionnaire. This questionnaire has 2 sections: demographic profile and a brief mental status examination. Those who received less than 20 were removed. According to this questionnaire who has no problem in navigation, registration, paying attention, calculation, remembering and drawing receive 30 scores and those with 25-30 have minor cognitive disorders. ${ }^{15}$

The statistical population of this study was composed of 40 elderly members referring to a Community Center in the city of Shiraz selected on a base of simple random sampling and divided into four random groups. Out of them, three groups participated in 90 -min group reminiscence sessions (once a week) for seven weeks in a row, while the members of one group as the control group did not attend any of the sessions. Each session took 30-35 min. Using the questionnaire developed by Schering, EI level among the individuals was examined in the form of pre-testpost-test. To test the hypotheses, the mean scores of the differences for pre-tests and post-tests of the experimental and control groups were evaluated through multivariate analysis of covariance.

EI scale was used to measure EI in this study. Since the options in this scale were set on a 5point Likert-type form, scoring was from 5 to 1 (strongly agree to strongly disagree) and in some items with negative content from 1 to 5 (strongly agree to strongly disagree). The total score of each scale and the sum of scores for the questionnaire were equal to the sum of the scores for 15 scales. The reliability of the questionnaire was calculated by 0.88 via split-half method and using Cronbach's alpha of 0.93 .

This study aimed at investigating the effects of group reminiscence therapy on EI in the elderly. The results of the research data using descriptive and inferential statistics were used to test the hypotheses.

\section{Results}

According to Table 1, differences in mean scores for pre-tests and post-tests on the variable of EI were 21.15 and -2 in the experimental and control groups, respectively. The levels of significance for all the variables according to Kolmogorov-Smirnov test were 0.652 and 0.747 respectively suggesting that the data followed a normal distribution and the use of parametric statistics was allowed to have an inferential analysis of data.

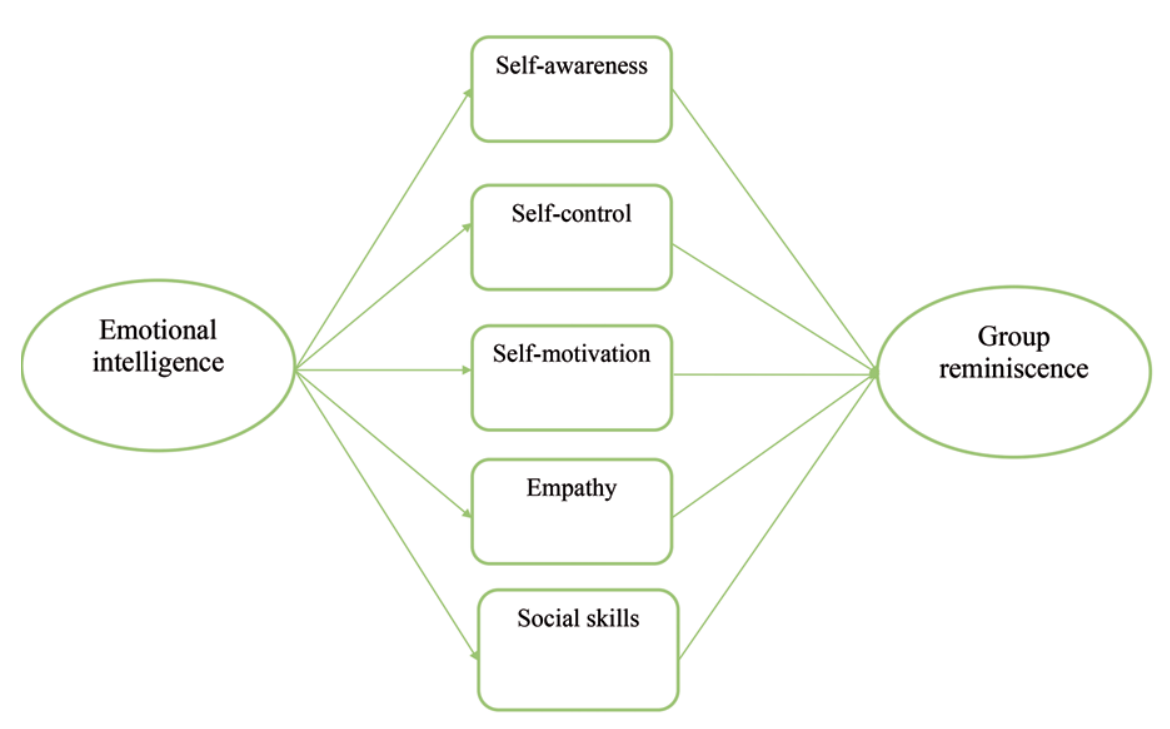

Figure 1. The conceptual model of the study. 


\section{Main hypothesis}

Interventions in the form of group reminiscence therapy have effects on emotional intelligence in the elderly.

The Levene's test results for the analysis of equal variances, with a $95 \%$ confidence level and with a measurement error of $\alpha=0.05$, showed a level of significance of 0.854 , suggesting that the difference of variances was not statistically significant and the assumption of equal variances was established.

As shown in Table 2, the analysis of covariance demonstrated that the mean scores of the EI variable for the post-test in the experimental group were significantly higher than those in the control group. This finding suggested that interventions in the form of group reminiscence had significant effects on EI in the elderly and considering Eta squared coefficient, the impact of group reminiscence on EI in the elderly was 0.644 .

\section{Hypothesis 1}

Interventions in the form of group reminiscence have significant effects on self-awareness in the elderly.

The Levene's test results for the analysis of equal variances, with a $95 \%$ confidence level and with a measurement error of $\alpha=0.05$, showed a level of significance of 0.338 , suggesting that the difference of variances was not statistically significant and the assumption of equal variances was established.

As shown in Table 3 the mean scores of the self-awareness variable for the post-test in the experimental group were significantly higher than those in the control group suggesting that interventions in the form of group reminiscence had significant effects on self-awareness in the elderly and considering Eta squared coefficient, the impact of group reminiscence on self-awareness in the elderly was 0.365 .

\section{Hypothesis 2}

Interventions in the form of group reminiscence have significant effects on self-control in the elderly.

As reported in Table 4, the mean scores of the self-control variable for the post-test in the experimental group were significantly greater than those in the control group suggesting that interventions in the form of group reminiscence had significant effects on self-control in the elderly and considering Eta squared coefficient, the impact of group reminiscence on self-control in the elderly was 0.396 .

\section{Hypothesis 3}

Interventions in the form of group reminiscence have significant effects on self-motivation in the elderly.

As showed in Table 5, the mean scores of the self-motivation variable for the post-test in the experimental group were significantly greater than those in the control group suggesting that interventions in the form of group reminiscence had significant effects on self-motivation in the elderly and considering Eta squared coefficient, the impact of group reminiscence on self-motivation in the older adults was 0.291.

\section{Hypothesis 4}

Interventions in the form of group reminiscence have significant effects on empathy in the elderly.

As reported in Table 6, the mean scores of the empathy variable for the post-test in the experimental group were significantly greater than those in the control group. This finding suggested that interventions in the form of group reminiscence had significant effects on empathy in the elderly and considering Eta squared coefficient, the impact of group reminiscence on empathy in the elderly was 0.391 .

\section{Hypothesis 5}

Interventions in the form of group reminiscence have significant effects on social skills in the elderly.

As showed in Table 7, the mean scores of the social skills variable for the post-test in the experimental group were significantly greater than those in the control group. The data suggested that interventions in the form of group reminiscence had significant effects on social skills in the elderly and considering Eta squared

Table 1. The means and standard deviation scores of emotional intelligence based on control and experimental groups.

\begin{tabular}{|c|c|c|c|c|c|c|}
\hline Variable & Group & Test type & Number & Mean & Standard deviation & $\begin{array}{l}\text { Differences between } \\
\text { pre-test-post-test means }\end{array}$ \\
\hline \multirow{2}{*}{ Emotional intelligence } & Experimental & $\begin{array}{l}\text { Pre-test } \\
\text { Post-test }\end{array}$ & $\begin{array}{l}20 \\
20\end{array}$ & $\begin{array}{l}109.35 \\
130.05\end{array}$ & $\begin{array}{c}9.08 \\
8.165\end{array}$ & 21.15 \\
\hline & Control & $\begin{array}{l}\text { Pre-test } \\
\text { Post-test }\end{array}$ & $\begin{array}{l}20 \\
20\end{array}$ & $\begin{array}{l}109.6 \\
107.6\end{array}$ & $\begin{array}{c}9.73 \\
6.643\end{array}$ & -2 \\
\hline
\end{tabular}

Table 2. Results of analysis of covariance for the main hypothesis.

\begin{tabular}{lcccccc} 
Source of variances & Sum of squares & Degree of freedom & Mean squares & F-statistic & Significance level & Eta squared \\
Pre-test & 233.495 & 1 & 233.495 & 3.085 & 0.087 & 0.077 \\
Group & 5068.694 & 1 & 5068.694 & 66.973 & 0.000 & 0.644 \\
Error & 2800.255 & 37 & 75.683 & - & - & - \\
Total & $572,849.000$ & 40 & - & - & - & - \\
\hline
\end{tabular}

Table 3. Results of analysis of covariance for the first hypothesis.

\begin{tabular}{lcccccc} 
Source of variances & Sum of squares & Degree of freedom & Mean squares & F-statistic & Significance level & Eta squared \\
Pre-test & 42.865 & 1 & 42.865 & 3.441 & 0.072 & 0.085 \\
Group & 265.243 & 1 & 265.243 & 21.294 & 0.000 \\
\hline Error & 460.885 & 37 & 12.456 & - & - & - \\
Total & $26,455.000$ & 40 & - & - & - \\
\hline
\end{tabular}


coefficient, the impact of group reminiscence on social skills in the elderly was 0.183 .

\section{Discussion and Conclusions}

The main objective of this study was to investigate the effects of group reminiscence therapy on EI in the elderly. The problem of this study was whether group reminiscence therapy had effects on EI in the elderly or not. To this end, a statistical population was selected and the scores of EI questionnaire on $\mathbf{4 0}$ elderly individuals were obtained based on study inclusion criteria. 40 of those with high scores of EI were placed into an experimental group and a control group through simple random sampling. In the first group (experimental group), the effects of group reminiscence therapy were studied; however, the second group (control group) did not receive any treatment.

The findings of this study showed that at a
$90 \%$ confidence level and with a measurement error of $\alpha=0.05$, the level of significance in the variable of the group was less than 0.05 and the research hypotheses i.e., the effects of interventions in the form of group reminiscence therapy on self-awareness, self-control, self-motivation, empathy, and social skills in the older adults were approved, that is the mean score of the variables for the post-test in the experimental group that were significantly higher than the scores in the control group.

As a whole, the results of the study indicated that group reminiscence therapy had effects on EI in the elderly. The result of another posttest, which was done after a month, confirmed the positive effects of interventions on EI level of the experimental group. These findings were consistent with the research findings by several studies. ${ }^{1,4,7,8,10}$

Aging is not a disease but a stage of the life cycle with its own issues. Today, transformation from a young society to an older one is a phenomenon in developing countries and com- pliance with this rapid process is in need of basic changes in health-related, cultural, social, and economic policies to overcome the problems caused by it and provide a good platform for growth and excellence in health and well-being of the older adults.

The results revealed that the review of memories of life can improve and boost memory in the elderly. Haslam and colleagues ${ }^{16}$ considered group reminiscence therapy as a means to improve and promote general health of the elderly living in nursing homes and believed that this approach improves memory in the elderly; promotes health-related, social, and economic functions, and consequently improves the general health of the residents in the nursing homes.

According to the research findings, it seemed that the application of the training program for EI and social skills during old age was an effective approach to reduce behavioral problems and emotional distress; and at the same time, it was good opportunity to increase

Table 4. Results of analysis of covariance for the second hypothesis.

\begin{tabular}{lcccccc} 
Source of variances & Sum of squares & Degree of freedom & Mean squares & F-statistic & Significance level & Eta squared \\
Pre-test & 2.723 & 1 & 2.723 & 0.267 & 0.267 & 0.007 \\
Group & 246.852 & 1 & 246.852 & 24.212 & 24.212 & 0.396 \\
\hline Error & 377.277 & 37 & 10.195 & - & - & - \\
Total & $27,209.000$ & 40 & - & - & - \\
\hline
\end{tabular}

Table 5. Results of analysis of covariance for the third hypothesis.

\begin{tabular}{lcccccc} 
Source of variances & Sum of squares & Degree of freedom & Mean squares & F-statistic & Significance level & Eta squared \\
Pre-test & 9.946 & 1 & 9.946 & 0.934 & 0.34 & 0.025 \\
Group & 161.877 & 1 & 161.877 & 15.203 & 0.000 & 0.291 \\
\hline Error & 393.954 & 37 & 10.647 & - & - & - \\
Total & $26,566.000$ & 40 & - & - & - \\
\hline
\end{tabular}

Table 6. Results of analysis of covariance for the fourth hypothesis.

\begin{tabular}{lcccccc} 
Source of variances & Sum of squares & Degree of freedom & Mean squares & F-statistic & Significance level & Eta squared \\
Pre-test & 0.583 & 1 & 0.583 & 0.062 & 0.805 & 0.002 \\
Group & 224.405 & 1 & 224.405 & 23.715 & 0.000 & 0.391 \\
\hline Error & 350.117 & 37 & 9.463 & - & - & - \\
Total & $17,638.00$ & 40 & - & - & - \\
\hline
\end{tabular}

Table 7. Results of analysis of covariance for fifth hypothesis.

\begin{tabular}{lcccccc} 
Source of variances & Sum of squares & Degree of freedom & Mean squares & F-statistic & Significance level & Eta squared \\
Pre-test & 15.481 & 1 & 15.481 & 1.517 & 0.226 & 0.039 \\
Group & 84.668 & 1 & 84.668 & 8.295 & 0.007 & 0.183 \\
\hline Error & 377.669 & 37 & 10.207 & - & - & - \\
Total & $13,615.000$ & 40 & - & - & - \\
\hline
\end{tabular}


social compatibility and improve learning in the elderly people.

Moreover, the results of this study showed that the experimental group obtained a better status after interventions compared with their status before intervention, which indicated the effects of group reminiscence on reducing depression and stress among the elderly. Group reminiscence improves the states of depression and stress in the elderly and helps them promote their mental health through creating the right atmosphere to stimulate and strengthen elderly individuals' memory.

With aging, cognitive disorders such as memory loss begin to emerge as a natural process. In the early stages, short-term memory starts to decay and slow down. Reminiscence is usually a conceptual method for reviewing life events and it is considered as a psychological process in person or in groups in which the philosophy behind arguments about past events and accidents is the stimulation of memory.

Akuama and colleagues ${ }^{17}$ considered group reminiscence as a conceptual method for reviewing life events, which could lead to improvements in cognitive and mental states such as depression through stimulating memory and strengthening emotional outcomes.

Reminiscence therapy, derived from Erickson, helps elderly review their past times; therefore, it helps these individuals solve the growth stage of personality consistency and get rid of frustration. Group reminiscence provides opportunities for people to experience their past, to have positive attitudes to themselves as well as the senders and receivers of the messages. In this way, the existing interpersonal interactions in group lead to an escape from social isolation and the formation of social behaviors. Through the clarification of the effects of group reminiscence on the enhancement of EI in the elderly, it can be concluded that this easy and low-cost technique can be simply used in all healthcare centers for the elderly or individually at home. It is also expected that this method might be welcomed by older adults, their families, and the members of the health team.

The inappropriateness of the place where the treatment sessions were held, personnel's improper and unprofessional conduct with the elderly, participant loss, and the low number of the sample size in the experimental group were of the limitations of this study. Training sessions to encourage the administrators and personnel of these centers in terms of the importance of psychological treatments and how to deal properly with the elderly before interventions can have significant effects on the results of further investigations.

\section{References}

1. Torkashvand A. The effect of group reminiscence on quality of life in the elderly. Tehran University of Science and Research; 2010.

2. Choy JC, Lou VW. Effectiveness of the modified instrumental reminiscence intervention on psychological well-being among community-dwelling Chinese older adults: a randomized controlled trial. Am J Geriat Psych 2016;24:60-9.

3. Sohrabi MB, Zolfaghari P, Mahdizade F, et al. Evaluation and comparison of cognitive state and depression in elderly admitted in sanitarium with elderly sited in personal home. Knowl Health 2008;3:27-31.

4. Simon SS, Yokomizo JE, Bottino CM. Cognitive intervention in amnestic mild cognitive impairment: a systematic review. Neurosci Biobehav Rev 2012;36:1163-78.

5. Blake M. Group reminiscence therapy for adults with dementia: a review. Br J Comm Nurs 2013;18:228-33.

6. Song D, Shen Q, Xu T-Z, Sun Q-H. Effects of group reminiscence on elderly depression: a meta-analysis. Int J Nurs Sci 2014; 1:416-22.
7. Wang JJ. Group reminiscence therapy for cognitive and affective function of demented elderly in Taiwan. Int J Geriatr Psych 2007;22:1235-40.

8. Aguirre E, Spector A, Hoe J, et al. Maintenance cognitive stimulation therapy (CST) for dementia: a single-blind, multicentre, randomized controlled trial of maintenance CST vs. CST for dementia. Trials 2010;11:46.

9. Boddey J, Hodgkins A. The importance of emotional intelligence in the management of a playwork setting. J Playwork Pract 2015;3:3.

10. Goleman D. Emotional intelligence. 10th Anniversary Edition; Why it can matter more than IQ. New York, NY: Bantam Books; 2006.

11. Bradbury T, Graves J. Emotional Intelligence (skills and tests). Translation: Ganji M. Tehran: Savalan Publications; 2006.

12. Sobhaninejad M, Yoozbashi A. Emotional intelligence and management in the organization. Tehran: Yastoroon Publications; 2009.

13. Sadegh M, Leila M, Seyyed B, Fallahi M. Investigating the effect of group therapy on depression level in the elderly residents of Kahrizak Charity Foundation. Ofhoghe Danesh J 2001;6:8-15. [Article in Persian]

14. Carter PJ. Test your emotional intelligence. London-Philadelphia: Kogan Page; 2011.

15. Wu CH, Yao G. Psychometric analysis of the short-form UCLA Loneliness Scale (ULS-8) in Taiwanese undergraduate students. Personal Individ Diff 2008;44:1762-71.

16. Haslam C, Haslam SA, Jetten J, et al. The social treatment: the benefits of group interventions in residential care settings. Psychol Aging 2010;25:157.

17. Akanuma K, Meguro K, Meguro M, et al. Improved social interaction and increased anterior cingulate metabolism after group reminiscence with reality orientation approach for vascular dementia. Psych Res Neuroimag 2011;192:183-7. 